Article

\title{
Design Optimisation of the Feeding System of a Novel Counter-Gravity Casting Process
}

\author{
Michail Papanikolaou ${ }^{1, *(\mathbb{D})}$, Emanuele Pagone ${ }^{1} \mathbb{( D )}$, Konstantinos Georgarakis ${ }^{1}$, Keith Rogers ${ }^{2} \mathbb{D}$, \\ Mark Jolly ${ }^{1}$ and Konstantinos Salonitis ${ }^{1}$ (D) \\ 1 Manufacturing Theme, Cranfield University, Cranfield MK 43 0AL, UK; e.pagone@cranfield.ac.uk (E.P.); \\ K.Georgarakis@cranfield.ac.uk (K.G.); m.r.jolly@cranfield.ac.uk (M.J.); k.salonitis@cranfield.ac.uk (K.S.) \\ 2 Cranfield Forensic Institute, Cranfield University, Cranfield MK 43 0AL, UK; k.d.rogers@cranfield.ac.uk \\ * Correspondence: m.papanikolaou@cranfield.ac.uk; Tel.: +44-(0)-1234-758235
}

Received: 31 August 2018; Accepted: 9 October 2018; Published: 11 October 2018

\begin{abstract}
The appropriate design of feeders in a rigging system is critical for ensuring efficient compensation for solidification shrinkage, thus eliminating (shrinkage-related) porosity and contributing to the production of superior quality castings. In this study, a multi-objective optimisation framework combined with Computational Fluid Dynamics (CFD) simulations has been introduced to investigate the effect of the feeders' geometry on shrinkage porosity aiming to optimise casting quality and yield for a novel counter-gravity casting process (CRIMSON). The weighted sum technique was employed to convert this multi-objective optimisation problem to a single objective one. Moreover, an evolutionary multi-objective optimisation algorithm (NSGA-II) has been applied to estimate the trade-off between the objective functions and support decision makers on selecting the optimum solution based on the desired properties of the final casting product and the process characteristics. This study is one of the first attempts to combine CFD simulations with multi-objective optimisation techniques in counter-gravity casting. The obtained results indicate the benefits of applying multi-objective optimisation techniques to casting processes.
\end{abstract}

Keywords: sand casting; counter-gravity; multi-objective optimisation; feeding system

\section{Introduction}

Metal casting is one of the oldest and most traditional manufacturing processes. It consists of a family of six processes, namely, melting, alloying, moulding, pouring, solidification, and finishing [1]. Heat treatment can also be added to the aforementioned processes as it facilitates obtaining the desired mechanical properties and relieving the residual stresses of the cast product [2]. Besides its complexity, casting is also characterised by its energy intensity. More specifically, a high amount of energy is demanded for the melting process, which accounts for about $55 \%$ of the overall energy consumption of the process [3]. Moreover, the resource efficiency of the casting family of processes is quite low. According to recent investigations, Operational Material Efficiency (OME), which is defined as the ratio between the mass of the shipped castings and the mass of the raw material used, was estimated to be as low as $27 \%$ [1]. It was found that about $50 \%$ of metal was chopped off in fettling and the need for carefully designing the running systems was clearly identified.

The Constrained Rapid Induction Melting Single Shot Up-Casting (CRIMSON) process, is a counter-gravity casting process designed to address the aforementioned issues related to material and energy efficiency [4]. During the CRIMSON process, the required quantity of metal to fill just a single mould is melted each time using superior quality raw materials (e.g., low concentration of impurities and dissolved gases). The molten metal is then introduced into the mould using a counter-gravity computer-controlled method. This process is characterised by: (a) higher energy 
efficiency compared to traditional methods, (b) superior yield, and (c) potential for higher quality products production by controlling the filling velocity throughout the duration of the process [5].

The quality and the performance of a casting product is strongly linked to defects, such as shrinkage, cold shots, and blowholes [6]. A few decades ago, the prediction of defective locations in castings relied mostly on the experience of the foundry engineers and human intuition. However, nowadays the evolution of the computing power and the advances in computational modelling have aroused considerable interest in the use of Computer Aided Engineering (CAE) which tends to replace the trial-and-error method in foundries. The simulation of casting processes involves a group of diverse phenomena including viscous and turbulent flow, phase change, various time scales and transient free boundaries. Since the mid 90's there have been significant efforts towards the development of software capable of accurately modelling the filling and solidification stages in casting [7]. Later on, computational tools for modelling casting defects, such as shrinkage microporosity [8], oxide film formation [9], and air entrainment at a free surface [10] were integrated into the existing simulation platforms.

These models were subsequently used by researchers for determining defective locations in castings by means of numerical modelling and for proposing optimal designs. Kwon et al. [11] modified the gate/runner system and the configuration of overflows of their High Pressure Die Casting (HPDC) setup to reduce porosities induced by air entrapment. Gawande et al. [12] performed design optimisation to minimise the residual stresses of a steel part induced during its solidification process using FEA. They performed multi-variable single-objective optimisation and proposed an optimal set of geometrical parameters minimising the maximum value of the residual stress. Their results were found to be in a good agreement with their experimental validation. An interesting optimisation case study has been performed by Sun et al. [13]. They optimised the geometrical parameters of a magnesium alloy casting gating system using the Taguchi method [14]. They performed multi-objective optimisation using scalarisation techniques to maximise the yield and simultaneously enhance the quality of their final product. Their optimal design parameters were different from case to case and dependent on the weights assigned to each objective function. The Taguchi method has also been employed by Dabade et al. [15], who optimised the gating and feeding system of a casting to reduce shrinkage porosity and increase the yield. A very interesting investigation combining the Design of Experiments method (DoE), training of Artificial Neural Networks (ANNs) and casting simulation software has been presented by Krimpenis et al. [16]. The purpose of this study was the optimisation of the die-casting process parameters such as the molten metal initial temperature to minimise the defect concentration. The Taguchi method was employed for selecting the minimum number of experiments to describe the dependency of their output variables on the process parameters. The output of their simulations was used as a database to successfully train a feedforward neural network with a mean relative error equal to $2.87 \%$ for the training set and $8.9 \%$ for the testing subset, respectively. Finally, the selection of the optimum die casting process parameters was performed using a Generic Algorithm (GA) fitness function.

CRIMSON is a casting process characterised by energy efficiency and superior quality cast products compared to traditional sand casting processes. However, the appropriate design of the rigging system is important to ensure the benefits of this novel process. The purpose of this study is the optimisation of the feeding system design with respect to the quality of the final casting product and the production yield. Computational Fluid Dynamics (CFD) simulations have been employed in conjunction with multi-objective optimisation algorithms. The results indicate that increasing the diameter and the height of the risers of the mould contributes towards higher quality casting products (less porosity) but on the other hand decreases the yield. To obtain the optimum solution of this multi-objective optimisation problem based on the importance of each component of the objective function the weighted sum method was employed. Finally, the trade-off between the final casting product quality, measured in terms of porosity, and the production yield was estimated and presented as a Pareto front using the Non-Dominated Sorting Genetic Algorithm-II (NSGA-II). 


\section{Simulation Methodology}

\subsection{CRIMSON}

As stated in the introduction, during the CRIMSON casting process molten metal is pushed in the mould with the help of a plunger using a counter-gravity computer-controlled method. A schematic illustration of the simulation setup is presented in Figure 1. Only one quarter of the simulation model was simulated by exploiting symmetry boundary conditions to reduce the computational cost [17]. For the filling and solidification simulations the commercial Flow-3D software (version 11.2) of Flow Science, Inc. (Santa Fe, NM, USA) [18] was used. The filling process was modelled using a mass source with a circular cross section located at the lower part of the central riser, which was later on removed during the solidification stage. The filling velocity was kept constant throughout the filling simulation, as the piston was assumed to maintain a constant-velocity, and was set equal to $0.12 \mathrm{~m} / \mathrm{s}$ so as to ensure that the maximum meniscus velocity at the thinner cross section of the tensile bars would not exceed $0.5 \mathrm{~m} / \mathrm{s}$ [19]. The wall boundary condition was considered to be no slip and the simulations performed accounted for both surface tension and turbulence (Renormalization Group model). For each simulation stage (filling and solidification) the fluid momentum and continuity equations were solved, while a first-order method was used for the approximation of the momentum advection. The simulation parameters are summarised in Table 1.

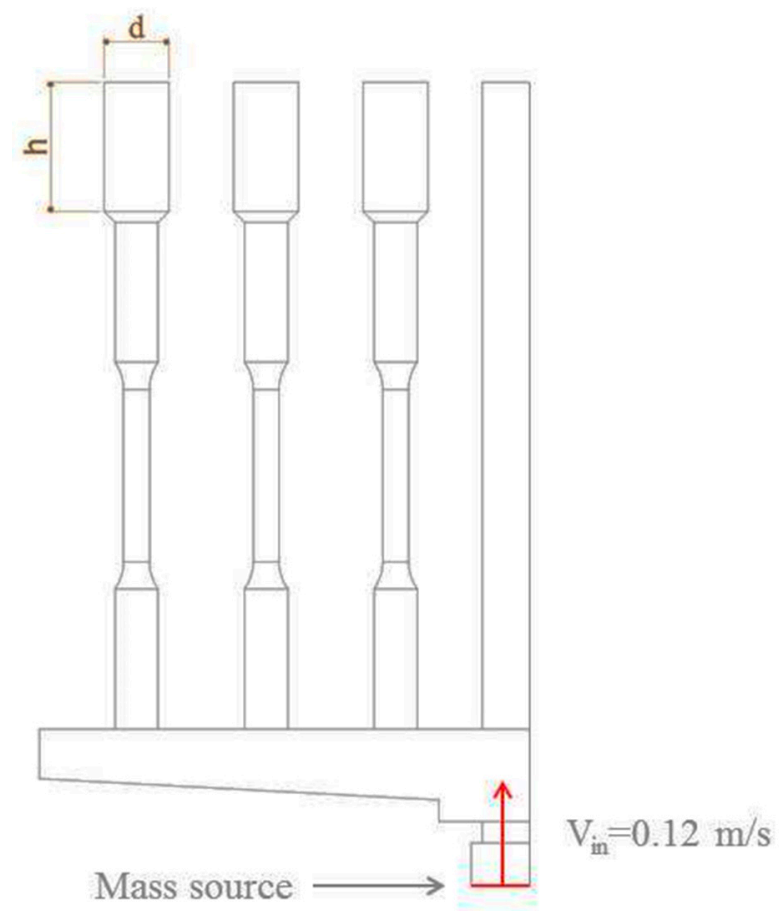

Figure 1. Simulation setup.

Using the Flow-3D materials database, the liquid metal was modelled as aluminium A356, while the mould component was modelled using the corresponding data for silica sand. In favour of accuracy, tabular data were used to describe the properties of the liquid metal, such as its solid fraction and viscosity, as a function of temperature. To estimate porosity, the macroporosity model in conjunction with the solidification with flow effects model of Flow-3D, were used.

An orthogonal mesh was applied to the simulation domain. The cell size was selected so as to ensure that at least 3 cells expand across the thinnest cross section of the casting ( 6 cells at the thinnest cross section). Symmetry boundary conditions were applied to all of the directions with the exception of $z_{\max }$, where a pressure boundary condition was used instead. 
Table 1. Simulation parameters.

\begin{tabular}{cc}
\hline Parameters & Values \\
\hline Mould surface temperature $\left({ }^{\circ} \mathrm{C}\right)$ & 25 \\
Pouring Temperature $\left({ }^{\circ} \mathrm{C}\right)$ & 700 \\
Air temperature $\left({ }^{\circ} \mathrm{C}\right)$ & 25 \\
Filling velocity $(\mathrm{m} / \mathrm{s})$ & 0.12 \\
Metal $/$ mould heat transfer coefficient $\left(\mathrm{W} /\left(\mathrm{m}^{2} \cdot \mathrm{K}\right)\right)$ & 1000 \\
Metal/air heat transfer coefficient $\left(\mathrm{W} /\left(\mathrm{m}^{2} \cdot \mathrm{K}\right)\right)$ & 30 \\
Sand thermal conductivity $(\mathrm{W} /(\mathrm{m} \cdot \mathrm{K}))$ & 0.7 \\
Surface tension coefficient $\left(\mathrm{kg} / \mathrm{s}^{2}\right)$ & 0.87 \\
\hline
\end{tabular}

\subsection{Optimisation}

Two input variables were used for the selected optimisation cases: (a) the diameter $(d)$ and (b) the height of the tensile bar risers $(h)$ as depicted in Figure 1. Moreover, the weighted sum method was implemented for determining the objective function of the multi-objective problem. This strategy converts the vector optimisation problem into a single-objective one by summing all of the individual objectives:

$$
\begin{gathered}
f(x)=\sum_{i=1}^{n} w_{i} f_{i}(x) \\
\sum_{i=1}^{n} w_{i}=1
\end{gathered}
$$

where $x=\left[\begin{array}{l}h \\ d\end{array}\right]$ and $w_{i}$ the weights corresponding to each component of the objective function. However, the riser dimensions should fall within a dimension range due to some constraints imposed from the current design. For instance, the riser diameter should always be smaller than $60 \mathrm{~mm}$ otherwise the risers would intersect each other. To satisfy the aforementioned design constraints imposed, the Constrained Optimization By Linear Approximation (COBYLA) [20] solver integrated in SciPY [21], was employed.

Besides providing the optimum solution for a specific set of weights it is of significant importance to illustrate the trade-off between the final product quality and yield. For this purpose, the Non-Dominated Sorted Algorithm-II [22], which is integrated in the Platypus optimisation framework [23], was used. NSGAs are evolutionary algorithms used to find a set of solutions with good convergence close to the Pareto optimal front. Finally, NSGA-II is an appropriate algorithm for this particular case study as it allows for bounded independent variables and consequently for constrained engineering applications.

\subsection{Validation of Numerical Modelling}

In order to validate the numerical model used in this study, preliminary results obtained from computed x-ray tomography scans (Nikon HMX ST 225 micro-CT, Nikon Metris X-Tek, Herts, UK) of aluminium A356 alloy tensile bars cast using the CRIMSON process (Figure 2a) were compared with complementary CFD simulations (Figure $2 b$ ). At this point, it should be noted that the counter-gravity cast tensile bar presented in Figure $2 \mathrm{a}$ has been produced without using optimised parameters for the CRIMSON technique, namely high quality raw material, rapid melting and appropriate feeding system, as a part of a systematic experimental evaluation of the parameters of the process. In this case, a small riser of $7.5 \mathrm{~mm}$ in height and $31 \mathrm{~mm}$ in diameter, insufficient to correctly compensate for the volume shrinkage during solidification, has been used. This has allowed for the evaluation of the porosity distribution along the sample. Nevertheless, the counter-gravity cast samples show relatively limited porosity, notably lower than the equivalent samples prepared with gravity-casting processes. Porosity is not distributed homogeneously across the sample; higher porosity can be observed at the 
top part of the tensile bar and the riser, indicating the insufficient feeding from the top riser, as expected. Interestingly, the simulated tensile bar (Figure $2 \mathrm{~b}$ ) captures adequately the porosity distribution across the sample and in particular the higher porosity at the riser area. A relatively good agreement is also found between the percentage porosity values obtained from the cast $(\sim 1.6 \%)$ and simulated samples $(1.22 \%)$ at the gage of the tensile bars. The slightly higher porosity in the cast samples is probably attributed to hydrogen induced gas porosity. Thus, the relatively good accord between experimental and simulated samples suggests the appropriateness of the numerical model and allows for the subsequent investigation of the effect of the riser geometry on the porosity of the castings. The results of the numerical simulations will be helpful for guiding the future design of the feeding system in the casting process.

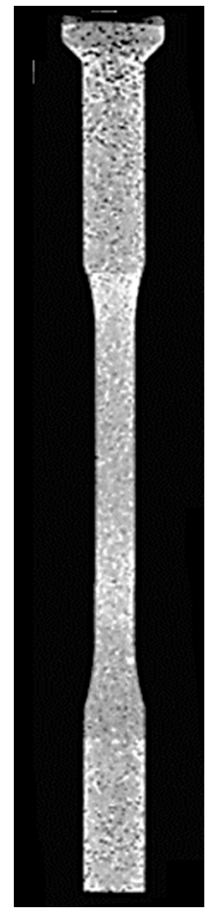

(a)

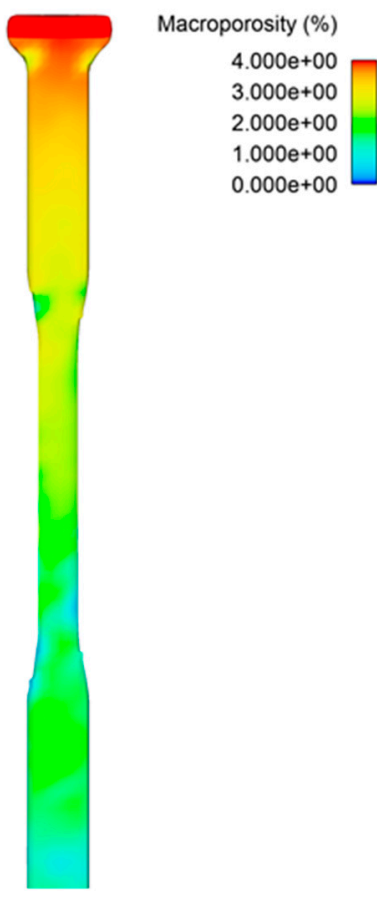

(b)

Figure 2. Macroporosity distribution across the top left tensile bar: (a) X-ray computed tomography and (b) CFD simulations.

\section{Results and Discussion}

\subsection{Feeder Design Effects on Macroporosity and Yield}

In order to measure the porosity of the final product the percentage macroporosity at the region of the tensile bars was spatially averaged at the end of each solidification simulation as illustrated in Figure 3. The macroporosity in the regions outside the interest area were not taken into account, as they are not part of the final product. As it can be observed in Figure 3, the increase of both the riser height and diameter contributes towards the reduction of macroporosity at the tensile bars area, as expected. In the cases Figure 3c,e where the riser diameter and riser height have obtained their maximum values respectively, the macroporosity in the tensile bars area has almost been eliminated. The reduction of macroporosity in the tensile bars region for larger values of the riser height and diameter is attributed to the enhanced feeding of this area due to higher pressure and the additional time required for the completion of the solidification of the risers. 
Macroporosity (\%)

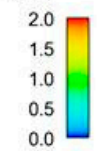

(a)

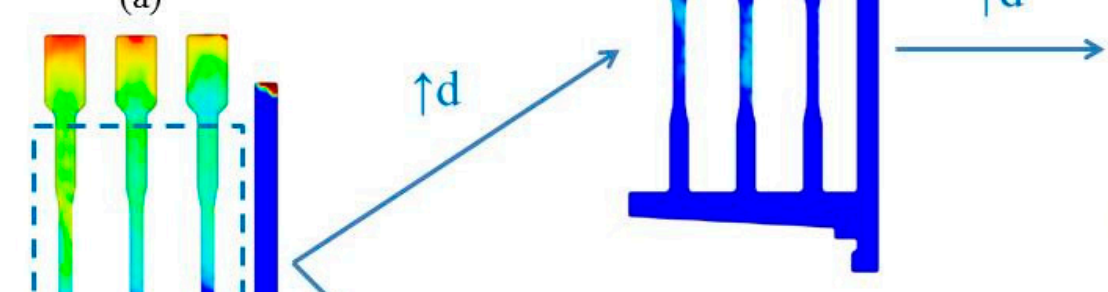

(d) (b)

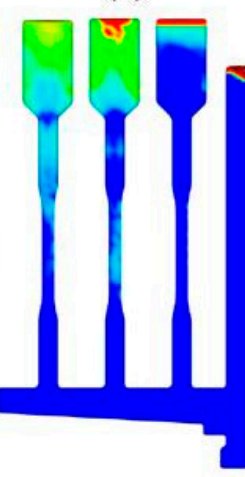

(c)

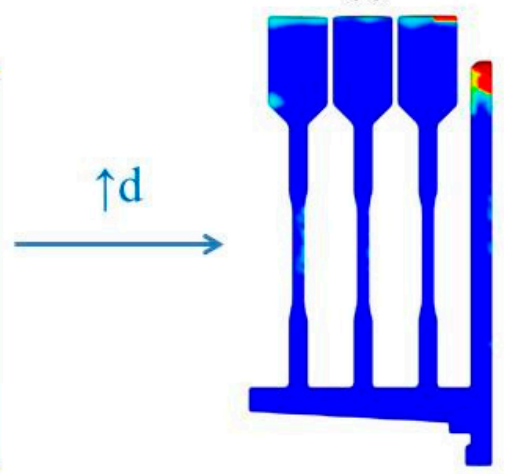

(e)

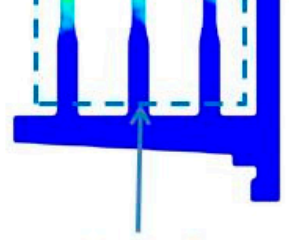

Area of

interest

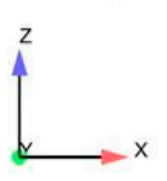

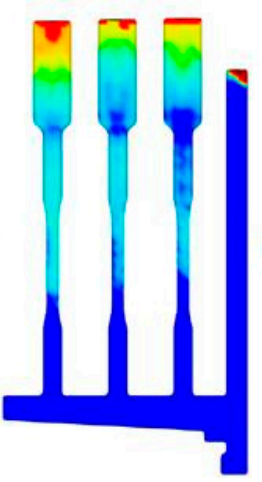

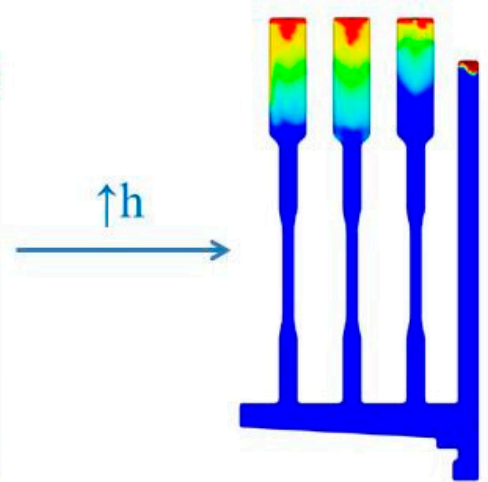

Figure 3. Effects of the riser dimensions on percentage macroporosity: (a) $h=49.41 \mathrm{~mm} / d=36 \mathrm{~mm}$; (b) $h=49.41 \mathrm{~mm} / d=46 \mathrm{~mm} ;$ (c) $h=49.41 \mathrm{~mm} / d=56 \mathrm{~mm} ;$ (d) $h=103.42 \mathrm{~mm} / d=36 \mathrm{~mm}$; and (e) $h=130.43 \mathrm{~mm} / d=36 \mathrm{~mm}$.

To investigate the effects of the riser design on macroporosity a set of 20 simulations for different combinations of the riser dimensions was performed. Porosity is lower compared to the results presented in Figure $2 \mathrm{~b}$ due to the larger riser dimensions, although its distribution remains similar. It can be observed that when the riser height exceeds $120 \mathrm{~mm}$ and the riser diameter remains greater than $36 \mathrm{~mm}$ the macroporosity observed in the tensile bars region is almost negligible. The same observation can be made for riser diameters greater than $51 \mathrm{~mm}$, provided that the riser height is always greater than $49.1 \mathrm{~mm}$. Therefore, it can be concluded that designing risers with dimensions higher than the ones proposed above will exclusively lead to lower energy efficiency and material waste. Moreover, it can be observed that some combinations of the variables under examination can also lead to reduced values of macroporosity. The optimal combination of design variables for optimum yield and product quality will be discussed in the following sections.

Figure $4 \mathrm{a}, \mathrm{b}$ shows how the riser dimensions affect porosity and demonstrate the potential for enhanced quality cast products. The reduction of porosity with increasing the risers' dimensions is partially attributed to the continuous flow of liquid metal which compensates for shrinkage. This is achieved via the top risers which act as a reservoir and solidify later that the cast part in line with the solidification geometrical modulus principle [19]. The effects of the riser dimensions on the solidification time are illustrated in Figure 4c,d. By comparing these two figures it can be observed that the riser diameter has a greater effect than the riser height on the solidification time. More specifically, the solidification time increases linearly with the riser height and approximately parabolically with the riser diameter. 
(a)

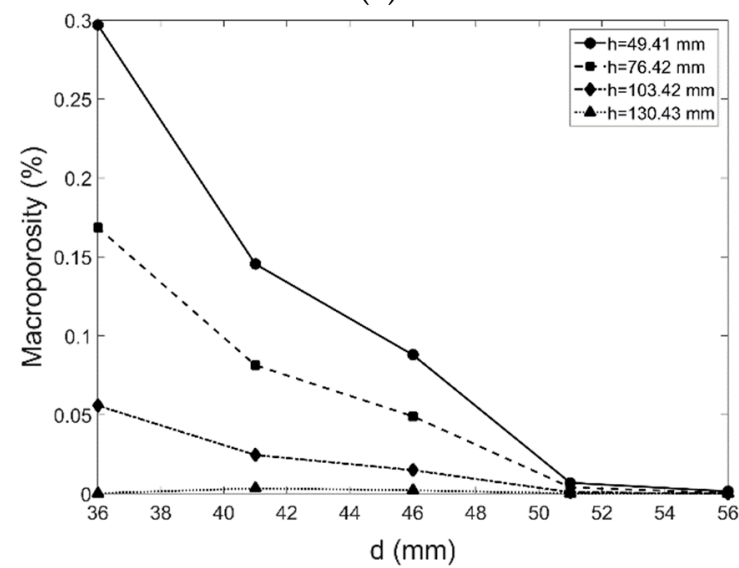

(c)

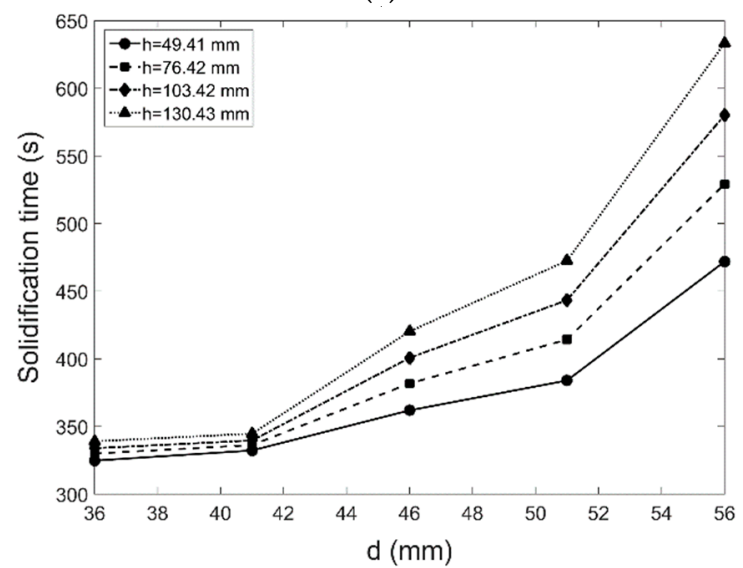

(b)

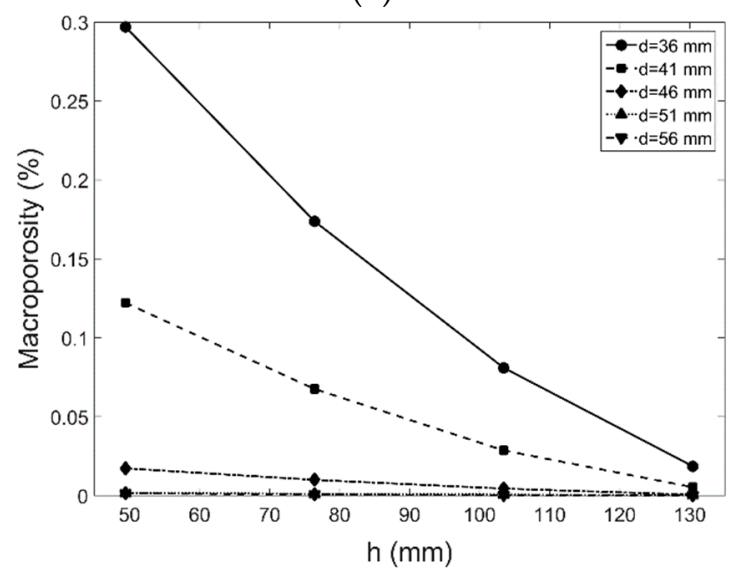

(d)

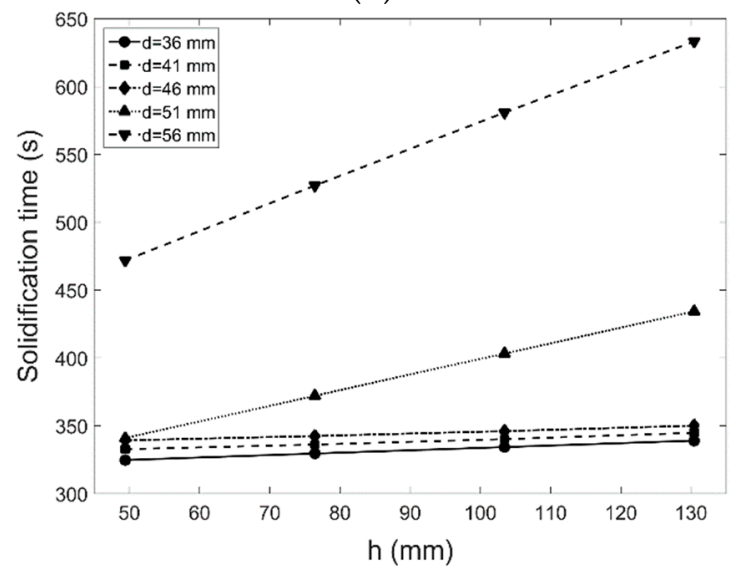

Figure 4. Percentage porosity plotted as a function of the (a) riser diameter and (b) riser height. Solidification time plotted as a function of the (c) riser diameter and (d) riser height.

This can be attributed to the fact that the volume of the tensile bars' risers is directly proportional to their height $h$ and the square of their radius $r=d / 2$ according to:

$$
V_{\text {Riser }}=h \cdot \pi\left(\frac{d}{2}\right)^{2}
$$

As expected, the increase of the riser dimensions and consequently the material volume has an impact on the yield, which has been evaluated as the ratio between the mass of the tensile bars and the total mass of the casting part. Thus, the desirable riser design should provide effective and sufficient feeding to compensate for shrinkage using the lowest possible material volume. Despite its relatively simple geometry, casting of tensile bars is characterised by certain challenges rather related to the smaller diameter in the middle of the bar (gage) than to the top and bottom parts [24]. This particular geometry dictates the need of having feeding action compensating for solidification shrinkage both from the top and bottom parts of the bars. Therefore, in such castings the yield is always relatively low. Previous work has shown that the yield of tensile bar castings using conventional gravity sand casting techniques is about $12 \%$, whereas the yield of the CRIMSON process can be twice as high [25]. The effect of the modified riser dimensions on the casting yield are illustrated in Figure 5 . The yield of the CRIMSON process has been normalised against the yield of conventional sand casting processes of tensile bars $(12 \%)$ and plotted against the riser diameter for various values of the riser height. It is evident that even with the modified riser dimensions the yield of the CRIMSON process can be as high as 1.7 times the yield of conventional sand casting processes. As expected, the increase of both the riser height and diameter deteriorate the yield performance of the CRIMSON casting process. This is 
because the mass of the risers increases while the mass of the tensile bars remains constant. It can be observed that in the case of $h=49.41 \mathrm{~mm}$ the yield drops by almost $35 \%$ as the riser diameter increases from $36 \mathrm{~mm}$ to $56 \mathrm{~mm}$. Similarly, for a constant value of the riser diameter $(d=36 \mathrm{~mm})$ the yield is reduced by $20 \%$ as the riser height rises from $49 \mathrm{~mm}$ to $130 \mathrm{~mm}$.

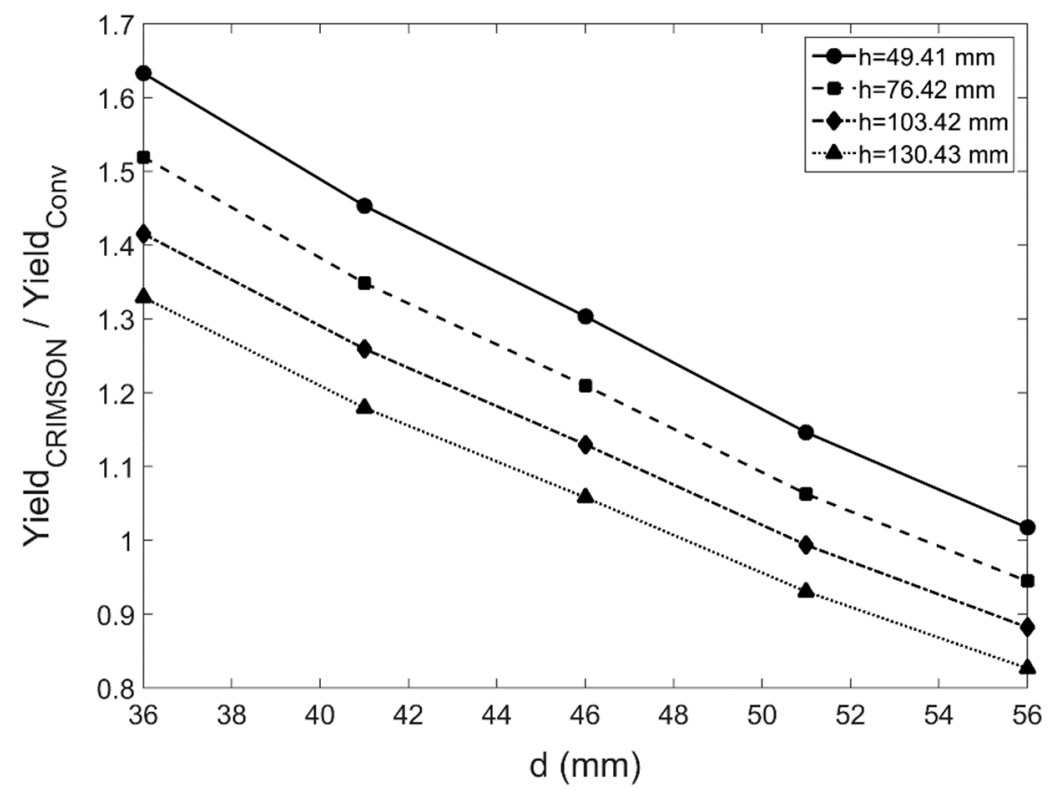

Figure 5. Normalised yield plotted as a function of the riser dimensions.

The results presented in this section provide a fair overview of the effects of the riser design on the final casting product quality and the efficiency of the process in terms of yield. However, at this point, a solid conclusion about the optimum combination of the riser dimensions leading to the desired result (minimisation of macroporosity and maximisation of yield), cannot be made. This topic falls into the scope of multi-objective optimisation and will be discussed in the following section.

\subsection{Optimisation}

To obtain the optimum combination of design parameters to improve both the quality and the yield of the CRIMSON process multi-objective optimisation techniques were used. One of the most common scalarisation techniques employed in multi-objective optimisation problems is the weighted sum method (Equation (1)). However, scalarisation might occasionally yield inaccurate results, especially when the different objective functions have different magnitude, as in this case. Therefore, each objective function $O F_{i}(x)$ was normalised by the difference of the optimal function values at the Nadir $\left(z^{N}\right)$ and Utopia points $\left(z^{U}\right)$ [26]. The optimisation problem can been defined as follows in mathematical terms:

$$
\begin{gathered}
\text { Minimise } f(x)=w_{M P} \cdot O F_{M P}+w_{Y} \cdot O F_{Y}=w_{M P} \cdot \frac{M P(x)-z_{M P}^{U}}{z_{M P}^{N}-z_{M P}^{U}}+w_{Y} \cdot \frac{Y(x)^{-1}-z_{Y}^{U}}{z_{Y}^{N}-z_{Y}^{U}} \\
\text { where: } \\
x=\left[\begin{array}{l}
h \\
d
\end{array}\right] \\
\text { subject to: } \\
49.41 \mathrm{~mm} \leq h \leq 130.43 \mathrm{~mm} \\
36 \mathrm{~mm} \leq d \leq 56 \mathrm{~mm} .
\end{gathered}
$$

The objective function has been evaluated by cubic spline interpolation over the two-dimensional grid of data in favour of computational efficiency and no additional simulations were performed. 
Whether an optimisation algorithm converges to a global minimum depends on the initial guess. For this purpose, 100 initial guesses were used and the three solutions corresponding to the minimum values of the objective function for each optimisation case study were estimated. Each proposed solution corresponds to a specific combination of weights as illustrated in Table 2. The global minimum for each optimisation case is denoted with bold. It can be observed that as the yield becomes an increasingly dominant optimisation criterion in place of macroporosity $\left(w_{M P} \rightarrow 0, w_{Y} \rightarrow 1\right)$, the average of the riser volume (calculated over the optimum solutions) decreases as expected. This is consistent with the results presented in the previous paragraph, which indicated that decreasing the riser dimensions leads to deteriorated quality but superior yield. Moreover, it can be seen that the values of the individual objective functions respond to the variation of the corresponding weights.

Table 2. Optimisation results.

\begin{tabular}{|c|c|c|c|c|c|c|c|c|}
\hline \multirow[b]{2}{*}{$w_{M P}$} & \multirow[b]{2}{*}{$w_{Y}$} & \multirow[b]{2}{*}{$\#$} & \multicolumn{6}{|c|}{ Solution } \\
\hline & & & $h(\mathrm{~mm})$ & $d(\mathrm{~mm})$ & $\begin{array}{c}\text { Average Riser } \\
\text { Volume }\left(\mathrm{m}^{3}\right)\end{array}$ & $\mathrm{OF}_{1}$ & $\mathrm{OF}_{2}$ & $f(x)$ \\
\hline \multirow{3}{*}{0.25} & \multirow{3}{*}{0.75} & 1 & 49.42 & 48.16 & \multirow{3}{*}{$5.07 \times 10^{-4}$} & $5.73 \times 10^{-3}$ & $8.74 \times 10^{-2}$ & $9.3 \times 10^{-2}$ \\
\hline & & 2 & 128.18 & 38.32 & & $2.28 \times 10^{-2}$ & $7.11 \times 10^{-2}$ & $9.4 \times 10^{-2}$ \\
\hline & & 3 & 118.84 & 39.03 & & $2.04 \times 10^{-2}$ & $7.45 \times 10^{-2}$ & $9.5 \times 10^{-2}$ \\
\hline \multirow{3}{*}{0.5} & \multirow{3}{*}{0.5} & 1 & 121.15 & 36 & \multirow{3}{*}{$4.68 \times 10^{-4}$} & $3.18 \times 10^{-2}$ & $1.05 \times 10^{-1}$ & $1.36 \times 10^{-1}$ \\
\hline & & 2 & 109.19 & 36.37 & & $6.07 \times 10^{-2}$ & $9.28 \times 10^{-2}$ & $1.54 \times 10^{-1}$ \\
\hline & & 3 & 108.28 & 36.63 & & $6.06 \times 10^{-2}$ & $9.52 \times 10^{-2}$ & $1.56 \times 10^{-1}$ \\
\hline \multirow{3}{*}{0.75} & \multirow{3}{*}{0.25} & 1 & 108.12 & 36 & \multirow{3}{*}{$3.98 \times 10^{-4}$} & $3.54 \times 10^{-2}$ & $1.28 \times 10^{-1}$ & $1.64 \times 10^{-1}$ \\
\hline & & 2 & 92.95 & 36 & & $7.88 \times 10^{-2}$ & $9.46 \times 10^{-2}$ & $1.73 \times 10^{-1}$ \\
\hline & & 3 & 92.5 & 36 & & $8.04 \times 10^{-2}$ & $9.36 \times 10^{-2}$ & $1.74 \times 10^{-1}$ \\
\hline
\end{tabular}

The weighted sum method is helpful in the case when the decision makers are aware of the values of the weights that should be assigned to each objective function. Moreover, the computational cost associated with this method is minimal. However, in the case of a non-convex objective function this method might not provide the true Pareto solutions [27]. This algorithm has to be repeated many times (by assigning various weights to the objective functions) in order to obtain an estimate of the Pareto frontier. When there is no intrinsic knowledge of the process under examination, the estimation of a Pareto frontier is of significant importance in order to illustrate the trade-off between the individual objective functions. Despite their computational cost (high number of function evaluations required), evolutionary algorithms are highly preferred for the estimation of Pareto frontiers and can produce multiple Pareto-optimal solutions with a single run.

In this investigation the NSGA-II algorithm [22] was used to demonstrate the trade-off between the quality of the final casting product and the yield (Figure 6). Each point of this Pareto front corresponds to a solution $x=\left[\begin{array}{l}h \\ d\end{array}\right]$ of the multi-objective problem. It can be observed that the yield decreases exponentially (or the inverse of yield increases exponentially) for lower macroporosity values. Based on the Pareto front of Figure 6, the decision maker can select the optimum combination of the riser design variables depending on the desirable characteristics of the casting process. By observing Figure 6, it can be concluded that increasing the riser dimensions beyond certain values is of minor benefit as macroporosity decays very slowly or obtains its minimum value, while there is a huge cost imposed on the efficiency of the process in terms of yield. Finally, an interesting topic for future investigation would be the integration of additional independent variables, such as time dependent filling velocity, in the current optimisation framework. 


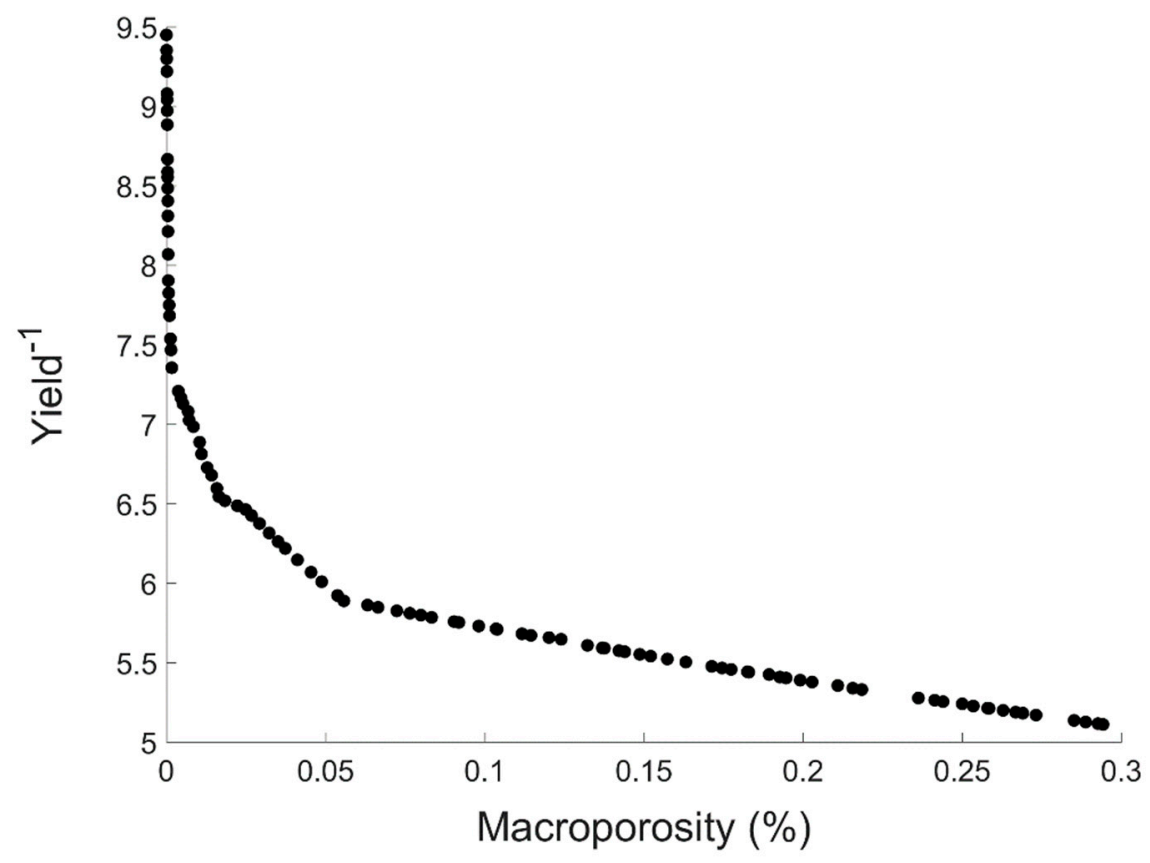

Figure 6. Pareto front of yield vs macroporosity.

\section{Conclusions}

In this investigation numerical simulations were performed to optimise the feeding system of the novel CRIMSON casting process. The objective of this optimisation case study has been the estimation of the optimum combination of the feeder height and diameter to maximise the cast product quality and the yield of the process. Initially, this multi-variable and multi-objective optimisation problem was transformed to a single objective one using the weighted sum method and a set of solutions, corresponding to different weight combinations using a linear programming method (COBYLA), was estimated. However, the weighted sum method requires an a priori knowledge of the weights that should be assigned to each objective function and in the case of non-convex functions does not produce Pareto optimal solutions. Therefore, the Non-dominated Sorting Genetic Algorithm II (NSGA-II) was employed to obtain an accurate estimation of the corresponding Pareto front and the trade-off between the quality and the yield/duration of the process was illustrated. The main conclusions of this investigation are summarised below:

- Weighted sum methods can be used to minimise the macroporosity and maximise the yield if there is a priori knowledge of the process characteristics and the weights that should be assigned to each of the objective function components. The optimisation result in this case is a set of riser dimensions $x=\left[\begin{array}{l}d \\ h\end{array}\right]$ which corresponds to a global minimum of the objective function for a specified set of weights.

- When there is no a priori knowledge of the process characteristics, the estimation of a Pareto front is highly suggested. The Pareto fronts of the macroporosity versus the yield/solidification time, drawn using the NSGA-II evolutionary algorithm, illustrate the trade-off between the aforementioned objective functions. It is evident that enhanced quality is offset by lower yield. Moreover, it has been shown that there is no need for increasing the riser dimensions beyond certain values as the final cast product macroporosity remains constant and equal to zero.

- The proposed optimisation framework contributes towards significant time savings as the total number of simulation runs is reduced by performing linear or cubic interpolation between an existing set of data points. Additional time savings can possibly be achieved by applying the Design of Experiments (DoE) method to estimate the minimum number of data points required to 
derive mathematical formulas accurately describing the dependence of the output variables on the independent ones.

- The multi-objective optimisation framework presented in this investigation has the potential to be applied on a wide span of casting processes and mould geometries. One of the reasons is that the adopted methodology allows for constrained independent variables which is the case in almost every engineering problem. Moreover, both the cases of a priori knowledge of the casting process characteristics and ab initio design are being covered. In the case where the objective function is a non-convex function, the NSGA-II algorithm can provide decision makers with a set of accurate solutions.

Author Contributions: Conceptualization, M.P., E.P., K.G., M.J. and K.S.; Investigation, M.P.; Methodology, M.P. and E.P.; Software, M.P. and E.P.; Supervision, K.G., M.J. and K.S.; Validation, K.G. and K.R.; Writing-original draft, M.P.; Writing—review \& editing, M.P., E.P., K.G., M.J. and K.S.; Funding Acquisition, Coordinated by M.J.

Funding: The authors would like to acknowledge the UK EPSRC projects "Small is Beautiful" and "Energy Resilient Manufacturing 2: Small is Beautiful Phase 2 (SIB2)" for funding this work under grants EP/M013863/1 and $\mathrm{EP} / \mathrm{P} 012272 / 1$ respectively.

Acknowledgments: The authors acknowledge J. Vian, B. Souchon and Y. Chao for experimental support.

Conflicts of Interest: The authors declare no conflict of interest.

\section{References}

1. Salonitis, K.; Zeng, B.; Mehrabi, H.A.; Jolly, M. The Challenges for Energy Efficient Casting Processes. Proced. CIRP 2016, 40, 24-29. [CrossRef]

2. Campbell, J. Complete Casting Handbook: Metal. Casting Processes, Techniques and Design, 2nd ed.; Elsevier: Amsterdam, The Netherlands, 2015; ISBN 978-0-444-63509-9.

3. BCS, Inc. Advanced Melting Technologies: Energy Saving Concepts and Opportunities for the Metal Casting Industry; BCS, Incorporated: Columbia, MD, USA, 2005.

4. Jolly, M.R. High integrity single shot casting process with reduced energy consumption. Mater. Technol. 2009, $24,143$.

5. Salonitis, K.; Jolly, M.R.; Zeng, B.; Mehrabi, H. Improvements in energy consumption and environmental impact by novel single shot melting process for casting. J. Clean. Prod. 2016, 137, 1532-1542. [CrossRef]

6. Couper, M.J.; Neeson, A.E.; Griffiths, J.R. Casting Defects and the Fatigue Behaviour of an Aluminium Casting Alloy. Fatigue Fract. Eng. Mater. Struct. 1990, 13, 213-227. [CrossRef]

7. Barkhudarov, M.R.; Hirt, C.W. Casting Simulation: Mold Filling and Solidification-Benchmark Calculations Using FLOW-3D ${ }^{\circledR}$; U.S. Department of Energy: Washington, DC, USA, 1995.

8. Hirt, C.W. Modeling Shrinkage Induced Micro-Porosity; Flow Science, Inc.: Santa Fe, NM, USA, 2004.

9. Reilly, C.; Green, N.R.; Jolly, M.R.; Gebelin, J.-C. The modelling of oxide film entrainment in casting systems using computational modelling. Appl. Math. Model. 2013, 37, 8451-8466. [CrossRef]

10. Hirt, C.W. Modeling Turbulent Entrainment of Air at a Free Surface; Flow Science, Inc.: Santa Fe, NM, USA, 2012.

11. Kwon, H.-J.; Kwon, H.-K. Computer aided engineering (CAE) simulation for the design optimization of gate system on high pressure die casting (HPDC) process. Rob. Comput. Integr. Manuf. 2018. [CrossRef]

12. Keste, A.A.; Gawande, S.H.; Sarkar, C. Design optimization of precision casting for residual stress reduction. J. Comput. Des. Eng. 2016, 3, 140-150. [CrossRef]

13. Sun, Z.; Hu, H.; Chen, X. Numerical optimization of gating system parameters for a magnesium alloy casting with multiple performance characteristics. J. Mater. Process. Technol. 2008, 199, 256-264. [CrossRef]

14. Roy, R.K. A Primer on the Taguchi Method; Society of Manufacturing Engineers: Dearborn, MI, USA, 2010; ISBN 0872638642.

15. Dabade, U.A.; Bhedasgaonkar, R.C. Casting Defect Analysis using Design of Experiments (DoE) and Computer Aided Casting Simulation Technique. Proced. CIRP 2013, 7, 616-621. [CrossRef]

16. Krimpenis, A.; Benardos, P.G.; Vosniakos, G.-C.; Koukouvitaki, A. Simulation-based selection of optimum pressure die-casting process parameters using neural nets and genetic algorithms. Int. J. Adv. Manuf. Technol. 2006, 27, 509-517. [CrossRef]

17. Taha, T.; Cui, Z.F. CFD modelling of slug flow in vertical tubes. Chem. Eng. Sci. 2006, 61, 676-687. [CrossRef] 
18. FLOW-3D CFD Software, Multiphysics Modeling, Numerical Simulation. Available online: https:/ / www. flow3d.com/ (accessed on 10 October 2018).

19. Campbell, J. Castings; Butterworth-Heinemann: Oxford, UK, 2003; ISBN 0080488447.

20. Buhmann, M.D.; Fletcher, R.; Barrodale, I.; Zala, C.; de Boor, C.; Micchelli, C.A.; Ron, A.; Conn, A.R.; Scheinberg, K.; Toint, P.L.; et al. Approximation Theory and Optimization: Tributes to M.J.D. Powell; Cambridge University Press: Cambridge, UK, 1997; ISBN 0521581907.

21. Minimize(method='SLSQP')—SciPy v1.1.0 Reference Guide. Available online: https:/ / docs.scipy.org/doc/ scipy/reference/optimize.minimize-slsqp.html (accessed on 10 October 2018).

22. Deb, K.; Pratap, A.; Agarwal, S.; Meyarivan, T. A fast and elitist multiobjective genetic algorithm: NSGA-II. IEEE Trans. Evol. Comput. 2002, 6, 182-197. [CrossRef]

23. Platypus-Multiobjective Optimization in Python-Platypus Documentation. Available online: http:// platypus.readthedocs.io/en/latest/ (accessed on 10 October 2018).

24. Gebelin, J. Simulation of Tensile Test Bars: Does the Filling Method Matter. In Simulation of Aluminum Shape Casting Processing: From Alloy Design to Mechanical Properties; Wiley: Hoboken, NJ, USA, 2006; pp. 299-310.

25. Zeng, B.; Salonitis, K.; Jolly, M.R. Comparison of the environmental impact of the CRIMSON process with normal sand casting process. In Proceedings of the 11th International Conference on Manufacturing Research: Advances in Manufacturing Technology XXVII, Cranfield, Bedfordshire, UK, 19-20 September 2013; pp. 281-286.

26. Grodzevich, O.; Romanko, O. Normalization and other topics in multi-objective optimization. In Proceedings of the Fields-MITACS Industrial Problems Workshop, Toronto, ON, Canada, 14-18 August 2006.

27. Strongin, R.G. Global Optimization with Non-Convex Constraints: Sequential and Parallel Algorithms; Springer: Berlin, Germany, 2000; ISBN 146154677X.

(C) 2018 by the authors. Licensee MDPI, Basel, Switzerland. This article is an open access article distributed under the terms and conditions of the Creative Commons Attribution (CC BY) license (http:/ / creativecommons.org/licenses/by/4.0/). 